\title{
CONVERGENCE OF AT-THE-MONEY IMPLIED VOLATILITIES TO THE SPOT VOLATILITY
}

\author{
VALDO DURRLEMAN, ${ }^{*}$ École Polytechnique
}

\begin{abstract}
We study the convergence of at-the-money implied volatilities to the spot volatility in a general model with a Brownian component and a jump component of finite variation. This result is a consequence of the robustness of the Black-Scholes formula and of the central limit theorem for martingales.
\end{abstract}

Keywords: Implied volatility; spot volatility; robustness formula; martingale central limit theorem

2000 Mathematics Subject Classification: Primary 91B70

Secondary 60F05; 60H05

\section{Introduction}

For a given traded option at time $t$ with strike $K$ and maturity $T$, the implied volatility is the unique volatility parameter $\Sigma_{t}(T, K)$ that must be put into the Black-Scholes formula to recover the option price. The implied volatility is therefore just another way of quoting option prices. In the case where the underlying security $S$ evolves like a geometric Brownian motion with deterministic volatility function $\sigma_{t}$,

$$
\frac{\mathrm{d} S_{t}}{S_{t}}=\sigma_{t} \mathrm{~d} W_{t},
$$

the implied volatility at time $t$ for an option with strike $K$ and maturity $T$ is

$$
\Sigma_{t}(T, K)=\sqrt{\frac{1}{T-t} \int_{t}^{T} \sigma_{s}^{2} \mathrm{~d} s} .
$$

Note that in this simple model the implied volatility does not depend on $K$. This formula has led most practioners and researchers in mathematical finance to think of the implied volatility as some kind of average of future values of the spot volatility, $\sigma$. In particular, it implies that the implied volatility converges to the spot volatility as we look at maturity dates $T$ that are closer and closer to today's date $t$. In practice, however, implied volatilities depend on the strike $K$ and the previous statement is thought to be true only for at-the-money options, i.e. for the strike $K=S_{t}$. This leads to the following fact: for every $t$,

$$
\lim _{T \downarrow t} \Sigma_{t}\left(T, S_{t}\right)=\sigma_{t} \quad \text { almost surely (a.s.). }
$$

This statement is often thought to be universally true, although no general proof has been proposed; see, for instance, [3], [5], [11], or [12]. It is also called the consistency condition

Received 30 July 2007; revision received 2 April 2008.

* Postal address: Centre de Mathématiques Appliquées, École Polytechnique - CNRS, Route de Saclay, 91128 Palaiseau, France. Email address: vdurrleman@gmail.com 
in [4]. In the case where the underlying asset is a continuous Markov process, it is a consequence of the more general result of [1]. Berestycki et al. [2] generalized [1] to the case where volatility is itself a diffusion; their results imply the above limit.

The present paper provides a simple proof of that fact that the above limit holds in a very general case where no Markov assumption is made and where jumps are allowed. In the presence of jumps it is not clear what we should call the spot volatility. Assuming the spot process is a locally square-integrable martingale, there are two possible candidates for the spot volatility: one based on $\langle S\rangle$ and one based on $\left\langle S^{\mathrm{c}}\right\rangle$, i.e. the predictable bracket of $S$ and that of its continuous part. We will see below that the right answer is given by the one based on $\left\langle S^{\mathrm{c}}\right\rangle$. This is quite surprising since it says that the effect of jumps cannot be seen in the short maturity limit of at-the-money implied volatilities. Let us point out that our results only apply when the jumps have finite variation.

Let us finally mention [6], which deals with the convergence of option prices to their intrinsic values as the option's time to maturity shrinks to 0 . Carr and $\mathrm{Wu}$ [6] showed that there exist different convergence speeds depending on whether or not jumps are present, the convergence speed depends on whether jumps have finite or infinite variation. While their paper is very interesting and shares common practical motivations with our paper, it should be stressed that looking at option prices instead of their implied volatilities is mathematically completely different.

In the next section we fix the framework and notation for the rest of the paper. In Section 3 we state and prove the main theorem (Theorem 1). Finally, in Section 4 we give the proofs of the auxiliary results needed in Section 3.

\section{Framework and notation}

Throughout this paper, we fix a probability space $(\Omega, \mathcal{F}, \mathrm{P})$ with a right continuous and P complete filtration $\left(\mathscr{F}_{t}\right)_{t \geq 0}$. We denote the conditional expectation given $\mathcal{F}_{t}$ by $\mathrm{E}_{t}\{\cdot\}$. We assume that on this probability space there exists an $\left(\mathcal{F}_{t}\right)_{t \geq 0} d$-dimensional Brownian motion $\left(\boldsymbol{W}_{t}\right)_{t \geq 0}$ and an integer random measure $j(\mathrm{~d} t \mathrm{~d} z)$ on $\mathbb{R}_{+} \times E$, where $E=\mathbb{R}^{e}$ for some integer $e$. We let $\bar{j}(\mathrm{~d} t \mathrm{~d} z)$ be its $\mathrm{P}$ compensator.

Let us consider a frictionless and arbitrage-free financial market where agents trade a stock $S$ and European calls (or, equivalently, puts) on $S$ with various maturities $T$ and strikes $K$. For simplicity, we assume that the risk-free interest rate is 0 and that the stock does not pay dividends. As is well known, these assumptions are equivalent to the assumption of deterministic interest rates and dividends. At short maturities, these have little effect on the prices of options. The absence of arbitrage implies the existence of a risk-neutral measure that prices all traded assets in a consistent way. Here, $\mathrm{P}$ is such a measure.

We fix a finite horizon $\mathcal{T}$. We will respectively denote by $S_{t}$ and $C_{t}(T, K)$ the spot price at $t$ of stock $S$ and the European call option price at $t$ on $S$ with strike $K$ and maturity $T$. Because of limited liability, $S_{t-}$ and $S_{t}$ are strictly positive.

Since $S$ is a local martingale, we let

$$
\frac{\mathrm{d} S_{t}}{S_{t-}}=\sigma_{t} \mathrm{~d} \boldsymbol{W}_{t}+\int_{E}\left(\exp \left(h_{t}(z)\right)-1\right)(j-\bar{j})(\mathrm{d} t \mathrm{~d} z) \quad \text { for } 0 \leq t \leq \mathcal{T},
$$

where $\sigma_{t}$ is the volatility vector and $h_{t}(z)$ is a predictable function on $\Omega \times \mathbb{R}_{+} \times E$ that models the jump sizes of the stock $S$. 
Using Itô's formula, we can rewrite $S$ as

$$
\begin{aligned}
\ln \frac{S_{t}}{S_{0}}= & \int_{0}^{t} \sigma_{s} \mathrm{~d} \boldsymbol{W}_{s}-\frac{1}{2} \int_{0}^{t} \sigma_{s}^{2} \mathrm{~d} s+\int_{0}^{t} \int_{E}\left(\exp \left(h_{s}(z)\right)-1\right)(j-\bar{j})(\mathrm{d} s \mathrm{~d} z) \\
& +\int_{0}^{t} \int_{E}\left(h_{s}(z)-\exp \left(h_{s}(z)\right)+1\right) j(\mathrm{~d} s \mathrm{~d} z) \quad \text { for } 0 \leq t \leq \mathcal{T}
\end{aligned}
$$

with $\sigma_{t}=\left|\sigma_{t}\right|$.

We now state our assumption on $S$.

Assumption 1. (a) The spot volatility $\sigma$ has right-continuous sample paths and there exist constants $\underline{\sigma}$ and $\bar{\sigma}$ such that, for every $t \in[0, \mathcal{T}]$,

$$
0<\underline{\sigma} \leq \sigma_{t} \leq \bar{\sigma} \quad \text { a.s. }
$$

(b) The compensator $\bar{j}$ has a density with respect to the Lebesgue measure $\bar{j}(\mathrm{~d} t \mathrm{~d} z)=$ $\bar{j}(t, z) \mathrm{d} t \mathrm{~d} z$ and there exists a constant $M$ such that, for every $t \in[0, \mathcal{T}]$,

$$
\int_{E}\left(\left|\exp \left(h_{t}(z)\right)-1\right|+1\right) \bar{j}(t, z) \mathrm{d} z<M \quad \text { a.s. }
$$

Assumption 1 has two simple but important consequences.

Lemma 1. (a) The stock $S$ is a uniformly integrable martingale on $[0, \mathcal{T}]$.

(b) The stock $S$ has finitely many jumps on $[0, \mathcal{T}]$.

Proof. (a) Follows from Lépingle and Mémin's criterion [10].

(b) Follows from the finiteness of $\int_{E}\left(\left|\exp \left(h_{t}(z)\right)-1\right| \wedge 1\right) \bar{j}(t, z) \mathrm{d} z$.

Let us now turn to the call price processes $C_{t}(T, K)$. First, we assume that, at each time $t$, all call options with strike $K>0$ and maturities $T>t$ are traded. Second, we assume that these are priced by the martingale measure $P$ :

$$
C_{t}(T, K)=\mathrm{E}_{t}\left\{\left(S_{T}-K\right)^{+}\right\},
$$

where $x^{+}=\max (0, x)$ for any real number $x$.

We can now define a unique implied volatility $\Sigma_{t}(T, K)$ to each call option price $C_{t}(T, K)$ by solving the equation

$$
C_{t}(T, K)=\mathscr{B} \&\left(t, S_{t}, T, K, \Sigma_{t}(T, K)\right),
$$

where $\mathcal{B} \&$ is the celebrated Black-Scholes formula:

$$
\mathscr{B} \&(t, S, T, K, \Sigma)=S \Phi\left(\frac{\ln (S / K)}{\Sigma \sqrt{T-t}}+\frac{\Sigma}{2} \sqrt{T-t}\right)-K \Phi\left(\frac{\ln (S / K)}{\Sigma \sqrt{T-t}}-\frac{\Sigma}{2} \sqrt{T-t}\right) .
$$

We denote by $\Phi$ the cumulative distribution functions of the standard Gaussian distribution.

We now state our very mild assumption on $C$ which ensures that the implied volatilities are finite and strictly positive.

Assumption 2. For every $t \in[0, \mathcal{T}], T>t$, and $K>0$,

$$
\left(S_{t}-K\right)^{+}<C_{t}(T, K)<S_{t} \quad \text { a.s. }
$$


At short maturities, option prices and implied volatilities have very specific behaviors which are consequences of (2).

Proposition 1. For every $t \in[0, \mathcal{T}]$ and $K>0$,

$$
\begin{gathered}
\lim _{T \downarrow t} \downarrow C_{t}(T, K)=\left(S_{t}-K\right)^{+} \quad \text { a.s., } \\
\lim _{T \downarrow t} \downarrow \Sigma_{t}(T, K) \sqrt{T-t}=0 \quad \text { a.s. }
\end{gathered}
$$

Proof. See Proposition 3 of [7].

\section{Statement and proof of the main result}

In this section we prove the main theorem of the paper, which is a much more precise result than (4).

Theorem 1. For every $t \in[0, \mathcal{T}]$,

$$
\lim _{T \downarrow t} \Sigma_{t}\left(T, S_{t}\right)=\sigma_{t} \quad \text { a.s. }
$$

The main ingredients for this theorem are the following two propositions. Proposition 2, below, is a generalization of the robustness formula of [8] to the case where jumps are present. Proposition 3, below, is the central limit theorem for martingales. Their proofs are postponed to Section 4.

Proposition 2. For every $t \in[0, \mathcal{T}], T>t$, and $K>0$, we have

$$
\begin{aligned}
& \frac{1}{2} \int_{0}^{1} \mathrm{E}_{t}\left\{\varphi\left(d_{1}\right) S_{t+\theta u}\left(\sigma_{t+\theta u}^{2}-\Sigma_{t}(T, K)^{2}\right)\right\} \frac{\mathrm{d} u}{\sqrt{1-u}} \\
& +\Sigma_{t}(T, K) \sqrt{T-t} \\
& \times \int_{0}^{1} \int_{E} \mathrm{E}_{t}\left\{\left(\mathcal{B} \&\left(t+\theta u, S_{t+\theta u} \exp \left(h_{t+\theta u}(z)\right), T, K, \Sigma_{t}(T, K)\right)\right.\right. \\
& \quad-\mathscr{B} \&\left(t+\theta u, S_{t+\theta u}, T, K, \Sigma_{t}(T, K)\right) \\
& \left.\left.\quad-\Phi\left(d_{1}\right) S_{t+\theta u}\left(\exp \left(h_{t+\theta u}(z)\right)-1\right)\right) \bar{j}(t+\theta u, z)\right\} \mathrm{d} z \mathrm{~d} u \\
& =0,
\end{aligned}
$$

where $\varphi$ denotes the density of the standard Gaussian distribution, $\theta=T-t$, and

$$
d_{1}=\frac{\ln \left(S_{t+\theta u} / K\right)}{\Sigma_{t}(T, K) \sqrt{\theta} \sqrt{1-u}}+\frac{1}{2} \Sigma_{t}(T, K) \sqrt{\theta} \sqrt{1-u} .
$$

Proposition 3. For every $t \in[0, \mathcal{T}], u \in[0,1]$, bounded $\mathcal{F}_{t}$-measurable random variable $Z$, and every bounded continuous function $f$,

$$
\lim _{\theta \downarrow 0} \mathrm{E}\left\{Z f\left(\frac{\ln \left(S_{t+\theta u} / S_{t}\right)}{\sqrt{\theta}}\right)\right\}=\mathrm{E}\{Z\} \mathrm{E}\left\{f\left(\sigma_{t} \sqrt{u} G\right)\right\},
$$

where $G$ is a standard Gaussian random variable independent of $\mathcal{F}_{t}$. 
Before we prove Theorem 1, we derive an easy consequence of Proposition 2 and we record it in the following lemma.

Lemma 2. For every $t \in[0, \mathcal{T}], T>t$, and $K>0$, we have

$$
\Sigma_{t}(T, K) \geq \underline{\sigma} \quad \text { a.s. }
$$

Proof. Because the function $S \mapsto \mathcal{B} \&(t, S, T, K, \sigma)$ is convex, the second term in (5) is nonnegative; hence,

$$
\int_{0}^{1} \mathrm{E}_{t}\left\{\varphi\left(d_{1}\right) S_{t+\theta u}\left(\sigma_{t+\theta u}^{2}-\Sigma_{t}(T, K)^{2}\right)\right\} \frac{\mathrm{d} u}{\sqrt{1-u}} \leq 0 .
$$

We let $A=\left\{\Sigma_{t}(T, K)<\underline{\sigma}\right\} \in \mathcal{F}_{t}$. Multipling the above inequality by $\mathbf{1}_{A}$ and taking the expectation, we obtain

$$
\mathrm{E} \int_{0}^{1} \mathrm{E}_{t}\left\{\mathbf{1}_{A} \varphi\left(d_{1}\right) S_{t+\theta u}\left(\sigma_{t+\theta u}^{2}-\Sigma_{t}(T, K)^{2}\right)\right\} \frac{\mathrm{d} u}{\sqrt{1-u}} \leq 0 .
$$

On $A, \sigma_{t+\theta u}^{2}-\Sigma_{t}(T, K)^{2}>\sigma_{t+\theta u}^{2}-\underline{\sigma}^{2} \geq 0$. Fubini's theorem yields $\mathrm{P}\{A\}=0$.

Proof of Theorem 1. First, we show that the triple integral involving the jumps in (5) has limit 0 in $L^{1}$ when $\theta$ goes to 0 . For every $S, \mathscr{B} \&(t, S, T, K, \sigma) \leq S$; therefore, the integrand is bounded by

$$
\begin{aligned}
& \mid \mathcal{B} \&\left(t+\theta u, S_{t+\theta u} \exp \left(h_{t+\theta u}(z)\right), T, K, \Sigma_{t}(T, K)\right)-\mathscr{B} \&\left(t+\theta u, S_{t+\theta u}, T, K, \Sigma_{t}(T, K)\right) \\
& -\Phi\left(d_{1}\right) S_{t+\theta u}\left(\exp \left(h_{t+\theta u}(z)\right)-1\right) \mid \\
& \quad \leq 2 S_{t+\theta u}\left(1+\left(\exp \left(h_{t+\theta u}(z)\right)-1\right)^{+}\right) .
\end{aligned}
$$

Therefore, by Assumption 1(b), the second term is bounded by $2 M S_{t} \Sigma_{t}(T, K) \sqrt{T-t}$, which, by (4), converges to 0 in $L^{1}$ by dominated convergence. Then, let $A \in \mathcal{F}_{t}$, multiply the first term in (5) with $K=S_{t}$ by $\mathbf{1}_{A}$, and take the expectation. This yields

$$
\lim _{T \downarrow t} \int_{0}^{1} \mathrm{E}\left\{\mathbf{1}_{A} \varphi\left(d_{1}\right) S_{t+\theta u}\left(\sigma_{t+\theta u}^{2}-\Sigma_{t}\left(T, S_{t}\right)^{2}\right)\right\} \frac{\mathrm{d} u}{\sqrt{1-u}}=0 .
$$

Now let $b_{t}=\lim \sup _{T \downarrow t} \Sigma_{t}\left(T, S_{t}\right)$. We take a sequence $T_{n}$ decreasing to $t$ such that $\lim _{n \rightarrow \infty} \Sigma_{t}\left(T_{n}, S_{t}\right)=b_{t}$. We also let $\theta_{n}=T_{n}-t$. We want to prove that $b_{t}<+\infty$ a.s., so by contradiction we assume that $b_{t}=+\infty$. Proposition 3 yields the fact that

$$
\frac{\ln \left(S_{t+\theta_{n} u} / S_{t}\right)}{\Sigma_{t}\left(T_{n}, S_{t}\right) \sqrt{\theta_{n}} \sqrt{1-u}}
$$

converges in law to 0 and, therefore, converges in probability to 0 for each $u \in[0,1)$. Thanks to (4), $d_{1}$ converges to 0 in probability for each $u \in[0,1)$. There is an $n$ after which the integrand in (6) along the subsequence $T_{n}$ is negative a.s. and for almost every (a.e.) $u$. Along a further subsequence, the integrand in (6) monotonically diverges to $-\infty$ a.s. and for a.e. $u$. By monotone convergence, we obtain the contradiction, since the left-hand side of (6) would be $-\infty$. 
Now let $a_{t}=\liminf _{T \downarrow t} \Sigma_{t}\left(T, S_{t}\right)$. We have $a_{t}>0$ by Lemma 2 and $a_{t}<+\infty$ since $b_{t}<+\infty$. We take a sequence $T_{n}$ decreasing to $t$ such that $\Sigma_{t}\left(T_{n}, S_{t}\right)$ decreases to $a_{t}$ as $n$ goes to $\infty$. We also let $\theta_{n}=T_{n}-t$ and rewrite the expectation above as a sum of four terms:

$$
\begin{aligned}
\int_{0}^{1} \mathrm{E}\left\{\mathbf{1}_{A} \varphi\left(d_{1}\right) S_{t+\theta_{n} u}\left(\sigma_{t+\theta_{n} u}^{2}-\Sigma_{t}\left(T_{n}, S_{t}\right)^{2}\right)\right\} \frac{\mathrm{d} u}{\sqrt{1-u}} \\
=\int_{0}^{1} \mathrm{E}\left\{\mathbf{1}_{A} \varphi\left(d_{1}\right) S_{t+\theta_{n} u}\left(\sigma_{t+\theta_{n} u}^{2}-\sigma_{t}^{2}\right)\right\} \frac{\mathrm{d} u}{\sqrt{1-u}} \\
\quad+\int_{0}^{1} \mathrm{E}\left\{\mathbf{1}_{A} \varphi\left(d_{1}\right)\left(S_{t+\theta_{n} u}-S_{t}\right)\left(\sigma_{t}^{2}-a_{t}^{2}\right)\right\} \frac{\mathrm{d} u}{\sqrt{1-u}} \\
+\int_{0}^{1} \mathrm{E}\left\{\mathbf{1}_{A} \varphi\left(d_{1}\right) S_{t+\theta_{n} u}\left(a_{t}^{2}-\Sigma_{t}\left(T_{n}, S_{t}\right)^{2}\right)\right\} \frac{\mathrm{d} u}{\sqrt{1-u}} \\
\quad+\int_{0}^{1} \mathrm{E}\left\{\mathbf{1}_{A} \varphi\left(d_{1}\right) S_{t}\left(\sigma_{t}^{2}-a_{t}^{2}\right)\right\} \frac{\mathrm{d} u}{\sqrt{1-u}} .
\end{aligned}
$$

We are now going to prove that the first, second, and third terms go to 0 . First fix $u \in[0,1]$ and note that

$$
\left|\mathbf{1}_{A} \varphi\left(d_{1}\right) S_{t+\theta_{n} u}\left(\sigma_{t+\theta_{n} u}^{2}-\sigma_{t}^{2}\right)\right| \leq S_{t+\theta_{n} u}\left|\sigma_{t+\theta_{n} u}^{2}-\sigma_{t}^{2}\right| \leq 2 \bar{\sigma}^{2} S_{t+\theta_{n} u} .
$$

The first inequality shows that the left-hand side converges to 0 a.s., while the second inequality shows that the left-hand side is a uniformly integrable sequence of random variables thanks to Lemma 1. Therefore,

$$
\lim _{n \rightarrow \infty} \mathrm{E}\left\{\left|\mathbf{1}_{A} \varphi\left(d_{1}\right) S_{t+\theta_{n} u}\left(\sigma_{t+\theta_{n} u}^{2}-\sigma_{t}^{2}\right)\right|\right\}=0 \quad \text { for each } u \in[0,1],
$$

by dominated convergence. The second inequality above implies that

$$
\frac{1}{\sqrt{1-u}} \mathrm{E}\left\{\left|\mathbf{1}_{A} \varphi\left(d_{1}\right) S_{t+\theta_{n} u}\left(\sigma_{t+\theta_{n} u}^{2}-\sigma_{t}^{2}\right)\right|\right\} \leq \frac{2 \bar{\sigma}^{2} S_{0}}{\sqrt{1-u}},
$$

and the conclusion follows by dominated convergence. Let us now consider the second and third terms with $A=\left\{\alpha<\sigma_{t}^{2}-a_{t}^{2}<\beta, S_{t}<\gamma\right.$, and $\left.\Sigma_{t}\left(T_{1}, S_{t}\right)^{2}-a_{t}^{2}<\delta\right\}$. The second term converges to 0 , since

$$
\left|\mathrm{E}\left\{\mathbf{1}_{A} \varphi\left(d_{1}\right)\left(S_{t+\theta_{n} u}-S_{t}\right)\left(\sigma_{t}^{2}-a_{t}^{2}\right)\right\}\right| \leq \max (\alpha, \beta) \mathrm{E}\left\{\left|S_{t+\theta_{n} u}-S_{t}\right|\right\} .
$$

The third term also converges to 0 by dominated convergence for any $\delta$. As for the fourth term, Proposition 3 yields, for every $u \in[0,1)$,

$$
\lim _{n \rightarrow \infty} \mathrm{E}\left\{\mathbf{1}_{A} \varphi\left(d_{1}\right) S_{t}\left(\sigma_{t}^{2}-a_{t}^{2}\right)\right\}=\mathrm{E}\left\{\mathbf{1}_{A} S_{t}\left(\sigma_{t}^{2}-a_{t}^{2}\right)\right\} \mathrm{E}\left\{\varphi\left(\frac{\sigma_{t} \sqrt{u} G}{a_{t} \sqrt{1-u}}\right)\right\} .
$$

The right-hand side is uniformly bounded in $u$; therefore, the dominated convergence theorem yields

$$
\mathrm{E}\left\{\mathbf{1}_{A} S_{t}\left(\sigma_{t}^{2}-a_{t}^{2}\right)\right\} \int_{0}^{1} \mathrm{E}\left\{\varphi\left(\frac{\sigma_{t} \sqrt{u} G}{a_{t} \sqrt{1-u}}\right)\right\} \frac{\mathrm{d} u}{\sqrt{1-u}}=0 .
$$

The integral in $u$ is strictly positive. Therefore, for every $\alpha, \beta, \gamma$, and $\delta, \mathrm{E}\left\{\mathbf{1}_{A} S_{t}\left(\sigma_{t}^{2}-a_{t}^{2}\right)\right\}=0$. This implies that $a_{t}=\sigma_{t}$ a.s.

A similar argument shows that $b_{t}=\sigma_{t}$ a.s., and the theorem is proved. 


\section{Proofs of Propositions 2 and 3}

Proof of Proposition 2. Itô's formula applied to $\mathcal{B} \&\left(s, S_{s}, T, K, \Sigma_{t}(T, K)\right)$ between $t$ and $T$ gives

$$
\begin{aligned}
& \left(S_{T}-K\right)^{+}-\mathcal{B} \&\left(t, S_{t}, T, K, \Sigma_{t}(T, K)\right) \\
& =\int_{t}^{T} \Phi\left(d_{1}^{-}\right) \mathrm{d} S_{s}+\int_{t}^{T}\left(\frac{1}{2} \frac{\varphi\left(d_{1}\right) S_{s}}{\Sigma_{t}(T, K) \sqrt{T-s}} \sigma_{s}^{2}+\frac{\partial \mathcal{B} \&}{\partial t}\left(s, S_{s}, T, K, \Sigma_{t}(T, K)\right)\right) \mathrm{d} s \\
& +\int_{t}^{T} \int_{E}\left(\mathcal{B} \&\left(s, S_{s-} \exp \left(h_{s}(z)\right), T, K, \Sigma_{t}(T, K)\right)-\mathscr{B} \&\left(s, S_{s-}, T, K, \Sigma_{t}(T, K)\right)\right. \\
& \left.-\Phi\left(d_{1}^{-}\right) S_{s-}\left(\exp \left(h_{s}(z)\right)-1\right)\right) j(\mathrm{~d} s \mathrm{~d} z)
\end{aligned}
$$

with

$$
d_{1}=\frac{\ln \left(S_{S} / K\right)}{\Sigma_{t}(T, K) \sqrt{T-s}}+\frac{1}{2} \Sigma_{t}(T, K) \sqrt{T-s}
$$

and

$$
d_{1}^{-}=\frac{\ln \left(S_{s-} / K\right)}{\Sigma_{t}(T, K) \sqrt{T-s}}+\frac{1}{2} \Sigma_{t}(T, K) \sqrt{T-s} .
$$

Now, since $(t, S) \mapsto \mathcal{B} \&\left(t, S, T, K, \Sigma_{t}(T, K)\right)$ solves the Black-Scholes partial differential equation,

$$
\left(\frac{\partial \mathcal{B} \&}{\partial t}+\frac{1}{2} \Sigma_{t}(T, K)^{2} S^{2} \frac{\partial^{2} \mathscr{B} \&}{\partial S^{2}}\right)\left(s, S, T, K, \Sigma_{t}(T, K)\right)=0,
$$

we have

$$
\begin{gathered}
\left(S_{T}-K\right)^{+}-\mathcal{B} \&\left(t, S_{t}, T, K, \Sigma_{t}(T, K)\right) \\
=\int_{t}^{T} \Phi\left(d_{1}^{-}\right) \mathrm{d} S_{s}+\int_{t}^{T} \frac{1}{2} \frac{\varphi\left(d_{1}\right) S_{s}}{\Sigma_{t}(T, K) \sqrt{T-s}}\left(\sigma_{s}^{2}-\Sigma_{t}(T, K)^{2}\right) \mathrm{d} s \\
\quad+\int_{t}^{T} \int_{E}\left(\mathcal{B} \&\left(s, S_{s-} \exp \left(h_{s}(z)\right), T, K, \Sigma_{t}(T, K)\right)-\mathscr{B} \&\left(s, S_{s-}, T, K, \Sigma_{t}(T, K)\right)\right. \\
\left.\quad-\Phi\left(d_{1}^{-}\right) S_{s-}\left(\exp \left(h_{s}(z)\right)-1\right)\right) j(\mathrm{~d} s \mathrm{~d} z) .
\end{gathered}
$$

Now we take conditional expectations given $\mathcal{F}_{t}$. Because of (2) and (3), the left-hand side of the above equation has conditional expectation 0 a.s. Since $S$ is uniformly integrable and $\Phi$ is a bounded function, the first term on the right-hand side also has expectation 0 a.s. Because the function $S \mapsto \mathcal{B} \&(t, S, T, K, \sigma)$ is convex, the last integrand in the above equation is nonnegative and, by definition of the compensator, we obtain

$$
\begin{aligned}
& \frac{1}{2} \mathrm{E}_{t} \int_{t}^{T} \frac{\varphi\left(d_{1}\right) S_{s}}{\Sigma_{t}(T, K) \sqrt{T-s}}\left(\sigma_{s}^{2}-\Sigma_{t}(T, K)^{2}\right) \mathrm{d} s \\
& +\mathrm{E}_{t} \int_{t}^{T} \int_{E}\left(\mathcal{B} \&\left(s, S_{s} \exp \left(h_{s}(z)\right), T, K, \Sigma_{t}(T, K)\right)-\mathscr{B} \&\left(s, S_{s}, T, K, \Sigma_{t}(T, K)\right)\right. \\
& \left.\quad-\Phi\left(d_{1}\right) S_{s}\left(\exp \left(h_{s}(z)\right)-1\right)\right) \bar{j}(s, z) \mathrm{d} z \mathrm{~d} s \\
& \quad=0 .
\end{aligned}
$$


Fubini's theorem is justified for the second integral, since the integrand is positive. To apply it to the first integral, we first break it into integrals with positive integrands. This is justified because

$$
\mathrm{E}_{t}\left\{\int_{t}^{T} \frac{\varphi\left(d_{1}\right) S_{s}}{\Sigma_{t}(T, K) \sqrt{T-s}} \Sigma_{t}(T, K)^{2} \mathrm{~d} s\right\} \leq \Sigma_{t}(T, K) \mathrm{E}_{t}\left\{\int_{t}^{T} \frac{S_{s}}{\sqrt{T-s}} \mathrm{~d} s\right\}<\infty,
$$

where the last inequality comes from Fubini's theorem and the martingale property. Making the change of variable $s=t+(T-t) u$ and multipling the result by $\Sigma_{t}(T, K) \sqrt{T-t}$ then completes the proof.

Proof of Proposition 3. Fix $t \in[0, \mathcal{T}]$. Define, for every $\theta>0$ and $u \in[0,1], g_{u}^{\theta}=\mathcal{F}_{t+\theta u}$ and $\tilde{S}_{u}^{\theta}=S_{t+\theta u}$. Then, $\tilde{S}^{\theta}$ is a family of semimartingales adapted to $\left(\mathcal{G}_{u}^{\theta}\right)_{u \in[0,1]}$. By time change,

$$
\begin{aligned}
\frac{\ln \left(\tilde{S}_{u}^{\theta} / \tilde{S}_{0}^{\theta}\right)}{\sqrt{\theta}}= & \int_{0}^{u} \tilde{\boldsymbol{\sigma}}_{r}^{\theta} \mathrm{d} \tilde{\boldsymbol{W}}_{r}^{\theta}-\frac{\sqrt{\theta}}{2} \int_{0}^{u}\left(\tilde{\sigma}_{r}^{\theta}\right)^{2} \mathrm{~d} r+\frac{1}{\sqrt{\theta}} \int_{0}^{u} \int_{E} \tilde{h}_{r}^{\theta}(z) \tilde{j}^{\theta}(\mathrm{d} r \mathrm{~d} z) \\
& -\sqrt{\theta} \int_{0}^{u} \int_{E}\left(\exp \left(\tilde{h}_{r}^{\theta}(z)\right)-1\right) \bar{j}(t+\theta r, z) \mathrm{d} r \mathrm{~d} z
\end{aligned}
$$

where $\tilde{\boldsymbol{W}}^{\theta}$ is a $\left(g_{u}^{\theta}\right)_{u \in[0,1]}$ Brownian motion, $\tilde{j}^{\theta}(\mathrm{d} r \mathrm{~d} z)$ is a jump measure adapted to $\left(g_{u}^{\theta}\right)_{u \in[0,1]}$, and $\theta \bar{j}(t+\theta r, z) \mathrm{d} r \mathrm{~d} z$ is its compensator. Finally, $\tilde{\boldsymbol{\sigma}}_{r}^{\theta}=\sigma_{t+\theta r}$ and $\tilde{h}_{r}^{\theta}(z)=h_{t+\theta r}(z)$.

Now, as $\theta$ goes to 0 , the first term in (7) converges $\mathcal{F}_{t}$ stably to a Gaussian random variable by an application of Theorem 5.42 of [9]. By Lebesgue's theorem, for each $u \in[0,1]$,

$$
\lim _{\theta \rightarrow 0}\left\langle\int_{0}^{\cdot} \tilde{\boldsymbol{\sigma}}_{r}^{\theta} \mathrm{d} \tilde{\boldsymbol{W}}_{r}\right\rangle_{u}=\lim _{\theta \rightarrow 0} \int_{0}^{u}\left(\tilde{\sigma}_{r}^{\theta}\right)^{2} \mathrm{~d} r=\sigma_{t}^{2} u \quad \text { a.s. }
$$

By Remark 5.55 of [9], the convergence is $\mathscr{F}_{t}$ mixing. This is the statement of the proposition. We only need to show that the three other terms in (7) converge to 0 a.s. The convergence to 0 of the second term is a consequence of Lebesgue's theorem. The convergence to 0 of the fourth term is also a consequence of Lebesgue's theorem and Assumption 1(b). As for the third term, the convergence to 0 is a result of the fact that the random measure $j^{\theta}$ has finitely many atoms for each $\theta>0$, so, for almost all $\omega$ and sufficiently small $\theta$,

$$
\int_{0}^{u} \int_{E} \tilde{h}_{r}^{\theta}(z) \tilde{j}^{\theta}(\mathrm{d} r \mathrm{~d} z)=0
$$

\section{Conclusion}

In this paper we considered a general martingale model for stock prices where jumps of finite variation are present. We proved the a.s. convergence of at-the-money implied volatilities to the spot volatility, more precisely, to the volatility of the continuous part of the stock model. An interesting open question is to understand what happens when jumps of infinite variation are present.

The present paper is a first step of a more general program that aims at computing implied volatilities in a generic martingale model. We think of Theorem 1 as the first term of a Taylor expansion of the implied volatility surface. In the continuous case, higher-order terms were computed in [7] by completely different means. 


\section{References}

[1] Berestycki, H., Busca, J. And Florent, I. (2002). Asymptotics and calibration of local volatility models. Quant. Finance 2, 61-69.

[2] Berestycki, H., Busca, J. And Florent, I. (2004). Computing the implied volatility in stochastic volatility models. Commun. Pure Appl. Math. 57, 1352-1373.

[3] Brace, A., Goldys, B., Klebaner, F. and Womersley, R. (2001). Market model for stochastic implied volatility with application to the BGM model. Tech. Rep., Department of Statistics, University of New South Wales.

[4] Carmona, R. (2007). HJM: a unified approach to dynamic models for fixed income, credit and equity markets. In Paris-Princeton Lectures on Mathematical Finance 2004 (Lecture Notes Math. 1919), Springer, Berlin, pp. $1-50$.

[5] CARR, P. (2000). A survey of preference free option valuation with stochastic volatility. Risk's 5th annual European derivatives and risk management congress, Paris.

[6] Carr, P. and Wu, L. (2003). What type of process underlies options? A simple robust test. J. Finance 58, 2581-2610.

[7] Durrleman, V. (2005). From implied to spot volatilities. Tech. Rep., Department of Mathematics, Stanford University. Available at http://math.stanford.edu/ valdo/papers/.

[8] El Karoui, N., Jeanblanc-Picqué, M. and Shreve, S. (1998). Robustness of the Black and Scholes formula. Math. Finance 8, 93-126.

[9] JACOD, J. AND Shiryaev, A. (2003). Limit Theorems for Stochastic Processes, 2nd edn. Springer, Berlin.

[10] LÉPIngle, D. AND MÉmin, J. (1978). Sur l'intégrabilité uniforme des martingales exponentielles. Z. Wahrscheinlichkeitsth. 42, 175-203.

[11] Medvedev, A. And Scaillet, O. (2007). Approximation and calibration of short-term implied volatilities under jump-diffusion stochastic volatility. Rev. Financial Studies 20, 427-459.

[12] Schönbucher, P. (1999). A market model for stochastic implied volatility. Philos. Trans. R. Soc. London Ser. A 357, 2071-2092. 